\title{
Field Cooling Magnetization and Losses of an Improved Architecture of Trapped-Field Superconducting Rotor for Aircraft Applications
}

\author{
V. Climente-Alarcon, ${ }^{1}$ A. Smara ${ }^{1}$, A. Patel, ${ }^{1}$ and B. A. Glowacki ${ }^{2}$ \\ University of Cambridge, Cambridge, UK \\ A. Baskys ${ }^{3}$ \\ CERN, Geneva, Switzerland \\ and \\ T. Reis ${ }^{4}$ \\ Oswald Elektromotoren GmbH, Miltenberg, Germany
}

\begin{abstract}
A hybrid electric configuration for aircraft propulsion can yield several advantages, reducing fuel consumption and take-off distance, improving control and decreasing emissions. For such a benign scenario to occur, advances destined to increase the power-to-weight ratio of actual electric motors must be developed. Superconducting technology offers the prospect of achieving such performance, but at the cost of increasing design and constructive complexity. In that sense, stacks consisting of piling up layers of high temperature superconductor have proven to trap high value current vortexes and thus can provide a source of magnetic flux density for torque production, without the need of current leads or other equipment in the rotor. However, these macroscopic currents must be induced prior to operation and then maintained undisturbed by any variation of the magnetic flux density in the airgap, which cause heating and demagnetization. This work presents the result of novel numerical computations on a new rotor architecture developed within the ASuMED project with the aim of facilitating the magnetization of the stacks from a superconducting stator and prevent their demagnetization during torque production. The performance of the machine is assessed, and the expected survivability of the stacks compared with laboratory measurements.
\end{abstract}

\section{Nomenclature}

$A \quad=$ Linear current density

$=$ Magnetic vector potential

$=$ Magnetic flux density

$=$ Electric field

$=$ Limit value of the electric field to be considered the material in superconducting state

$=$ Magnetic field

$=$ Current density

$=$ Critical current (maximum DC current circulating in a superconductor before quenching)

\footnotetext{
${ }^{1}$ Research Associate, Dpt. of Materials Science and Metallurgy, University of Cambridge, CB3 0FS, Cambridge, UK ${ }^{2}$ Professor, Department of Materials Science and Metallurgy, University of Cambridge, CB3 0FS, Cambridge, UK and also Professor of Energy at Institute of Power Engineering, Mory 8, 01-330 Warsaw, Poland.

${ }^{3}$ Research Associate, CERN - Conseil Européen pour la Recherche Nucléaire, Espl. des Particules 1, 1217 Meyrin, Switzerland

${ }^{4}$ Project Manager, Oswald Elektromotoren GmbH, Benzstraße 12, 63897 Miltenberg, Germany
} 


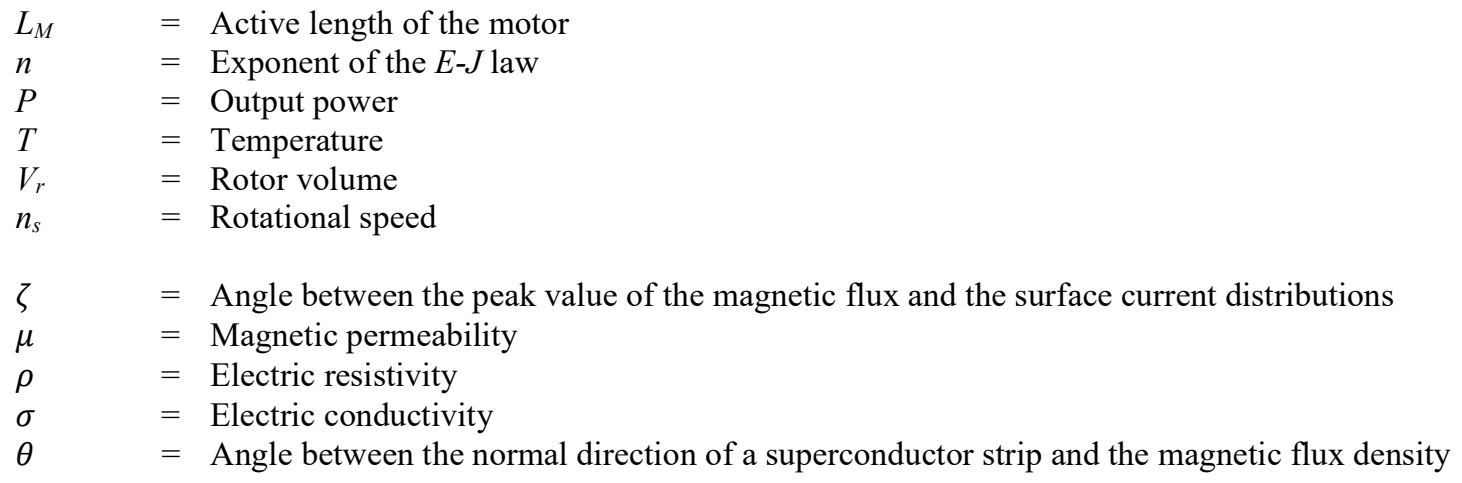

\section{Introduction}

Hybrid electric technology has been agreed as an answer to meet the future demands for aircraft propulsion [1]. Under this architecture the speed ratio between of the fan and the core of a turbofan motor is bridged by using a motorgenerator arrangement equivalent to an electric transmission. Furthermore, once divided propulsion and electricity generation, the corresponding elements can be installed in the most aerodynamic favorable portions on the aircraft for each one, allowing, for instance, configurations that improve propulsion efficiency by ingesting the boundary layer, as proposed in [2]. Nevertheless, to make such configuration feasible, the power density of actual electrical motors must be increased one order of magnitude from the actual state of the art designs, $5 \mathrm{~kW} / \mathrm{kg}$ [3], up to $40 \mathrm{~kW} / \mathrm{kg}$.

In the early stages of design of an electrical motor, the power density (proportional to the ratio $P / V r$ where $P$ is yielded power and $V_{r}$ the volume of the rotor) is approximated as:

$$
\frac{P}{V_{r}}=n_{s} \hat{A} \hat{B} \cos \zeta
$$

where $\hat{A}$ is the peak value of the surface stator current, $\hat{B}$ is the peak value of the magnetic flux density -both considered sinusoidal-, $\zeta$ the angle between both distributions, and $n_{s}$ is the rotational speed [4]. Some advances in increasing power density have been achieved by rising $n_{s}$, that is, by repeating the same cycle at a faster rate; however, simply acknowledging the intended application of this kind of machine, moving the fan of a turbopropulsor at a slow speed, would exclude this way of proceeding. Hence, a low speed/very high torque electrical motor is needed for such function -which constitutes a highly demanding design- and the way of achieving is by either acting on the currents of the stator $\hat{A}$, the flux produced by the rotor $\hat{B}$ or, given the sharp increase in performance needed, preferably on both.

Superconductive technology may be an enabler in this sense [5]. Through decades of manufacturing improvements high temperature superconductor materials can nowadays carry hundreds of amperes through conductors with cross sections below $0.1 \mathrm{~mm}^{2}$, even in the presence of an external magnetic flux. The use of high temperature superconductors in the stator winding of an electrical machine would multiply the current capacity there acting on $\hat{A}$ in (1). A similar arrangement was tried relatively early in the rotor of synchronous machines [6], since in principle the electromagnetic conditions there are more benign, increasing in this case $\hat{B}$ in (1). However, a rotor featuring superconductive windings is an element that from the mechanical point of view, must withstand a noticeable shrinkage during cooling, requiring the corresponding clearance in the bearings; and, from the thermal point of view, an internal circuit for refrigeration down to $10-20 \mathrm{~K}$ along with the corresponding thermal insulating couplings, therefore, adding a secondary electrical circuit with coils and feeders increases its complexity to levels that cast a doubt on its actual applicability.

In consequence, we proposed for the ASuMED project, aimed at developing a fully superconducting motor demonstrator for aircraft propulsion application [7], thus simplifying the rotor construction by eliminating the use of current leads or any feed to the rotor field winding. This is done by utilizing an arrangement similar to the one employed in permanent magnet motors. In this case an assembly of high temperature superconducting tapes, cut and piled up together, can be magnetized and maintain a flowing macroscopic current in the form of a vortex, so long as refrigeration is provided and no variation of the magnetic flux density occurs (Fig. 1). This solution is structurally sound, as the tape exhibits the mechanical properties of the substrate on which the superconductor is deposited, usually a superalloy. Unlike bulks they can be easily manufactured, being the defect present in any layer smoothed out by the 
others, and it is even possible to bend it to adapt to different shapes [8]. Such characteristics enabled achieving a record of $17.7 \mathrm{~T}$ trapped by a hybrid configuration of one of such stacks [9].

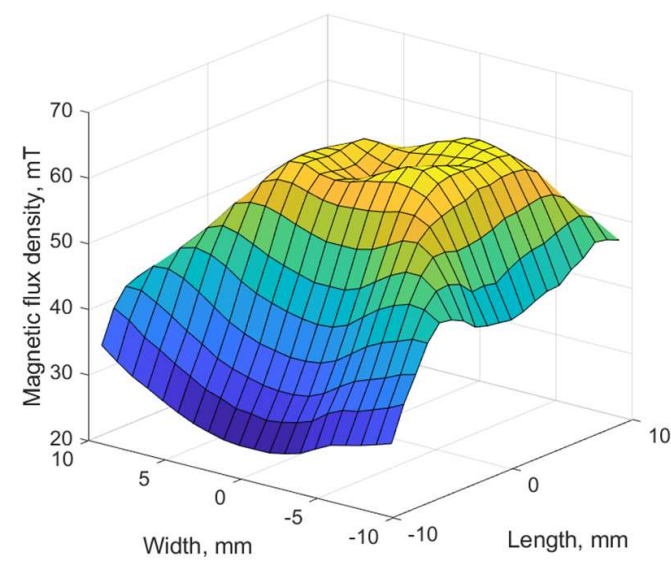

Fig. 1 Magnetic flux density trapped at the center of two layers of AMSC tape $(45 \times 45 \mathrm{~mm})$ for a temperature of $77 \mathrm{~K}$. This minimal stack is not fully saturated, as the cut-off summit indicates (max. value 62.6 mT).

Nevertheless, two important problems arise from the application of trapped-field magnets as the source of magnetic flux density in an electrical machine. First, the supercurrents must be induced prior to operation, and second, any magnetic flux variation will induce an electrical field that interacts with the macroscopic currents within the stack, yielding heat losses if the flux is normal to its surface [10], or demagnetization [11], if the flux is parallel. These kinds of variations are inherent to the airgap of an electrical machine due to the discrete distribution of stator coils and slots.

Two approaches are followed in the industry to avoid the influence of these harmonics in a conventional rotor (which when acting on permanent magnets cause heating and weakening of the remanent flux): either covering the rotor with a conductive sleeve, which insulates the rotor interior from high frequency components, or burying the magnets inside the rotor iron, where the magnetic environment features almost only the constant (DC) component. However, for a superconducting machine the first solution generates extra heat, that must be retrieved by the rotor cooling system at extreme cryogenic temperatures [12], whereas the second solution greatly hinders the magnetization of buried (interior mounted) superconducting stacks from a superconducting stator, since the leakage paths the rotor iron adds around the stacks subtract much of the flux, weakening it to low levels $[4,13]$, and pulsing is in principle not possible.

These conflicting requirements were identified early in the project [14] and in this work the solution devised is presented with the assistance of a 2D finite element model, developed by KIT also within ASuMED [15], weakly coupling two formulations. The model allows a superconducting machine to be treated roughly as a conventional one during this stage of its development and yields numerical results both about its performance (torque) and the expected losses in the stacks, providing insight about its demagnetization rate. Losses and demagnetization are compared to early assumptions and some laboratory measurements, respectively, which assess the applicability of the proposed design. For this purpose, the remainder of this work is organized as follows: Section III introduces the main architecture of the ASuMED motor, including the novel rotor lay-out developed; Section IV summarizes the finite element formulation used for simulating the superconducting stacks during magnetization and operation inside the machine [15]. Section V yields the results in two points: magnetization and operation; and finally, Section VI presents the conclusions.

\section{Motor Architecture}

The final configuration of the fully superconductive ASuMED motor features a classical electromagnetic radial construction with distributed winding; a double cryostat thermal configuration [16], with independent cooling for the stator and rotor, and mechanically -besides several solutions departing from conventional machines to accommodate for the almost $300 \mathrm{~K}$ of temperature reduction needed for operation-a teethless construction where the stator windings must be supported by a non-magnetic structure, thus not depicted in these simulations. 
As stated, stacks of superconductive tape are used in the role of permanent magnets to simplify the rotor construction. This is an element of great complexity due to the provision of a helium cooling system circulating between an internal fixed insert and the external moving part of the rotor [16]. This external part provides the mechanical attachment for the electromagnetic-active part of the rotor, is assumed to be maintained at $25 \mathrm{~K}$ during the operation of the machine. The characteristics of the simulated motor are presented in Table I.

\section{TABLE I}

\section{MOTOR CHARACTERISTICS}

\begin{tabular}{|l|c|lc|}
\hline Stator external diameter & $236 \mathrm{~mm}$ & Number of phases & 3 \\
\hline Stator iron internal diameter & $214 \mathrm{~mm}$ & Number of pole pairs & 5 \\
\hline Stator winding distance to center & $101.5 \mathrm{~mm}$ & Number of coil sides per pole and phase & 1 \\
\hline Stator coil cross section & $4 \times 10 \mathrm{~mm}$ & Stator turns per coil & 7 \\
\hline Stack height & $5 \mathrm{~mm}$ & Supply frequency & $500 \mathrm{~Hz}$ \\
\hline Rotor yoke external diameter & $190 \mathrm{~mm}$ & Operating stator current (RMS) & $400 \mathrm{~A}$ \\
\hline Rotor yoke internal diameter & $150 \mathrm{~mm}$ & Magnetizing stator current (DC) & $1,600 \mathrm{~A}$ \\
\hline Machine length & $175 \mathrm{~mm}$ & Torque & $500 \mathrm{~N} \cdot \mathrm{m}$ \\
\hline
\end{tabular}

As for the stator, the solution devised for the rotor departs from existing ones. Initially we considered the common configurations of surface mounted (Fig. 2 a)) and internal mounted stacks (Fig. 2 b)). The results of conventional 2D finite element computations in magnetization and operation conditions for the machine are shown in Table II. Clearly a higher peak magnetic flux density and flux per pole can be obtained with surface mounted stacks, but the normal and tangential variations of the magnetic flux density during operation are quite big despite the suppression of teeth in the stator, with losses rising as the third power of the field variation [10]. Interior mounted stacks are particularly well protected against the tangential variations that cause demagnetization, with values one order of magnitude below the previous ones, but their magnetization in the conditions simulated is poor, with a peak remanent flux and flux linked to the stator half or even lower than the first configuration due to the presence of the iron bridges necessary to fix the pole section in its place.

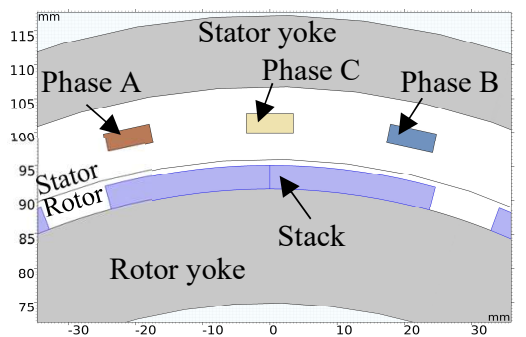

a)

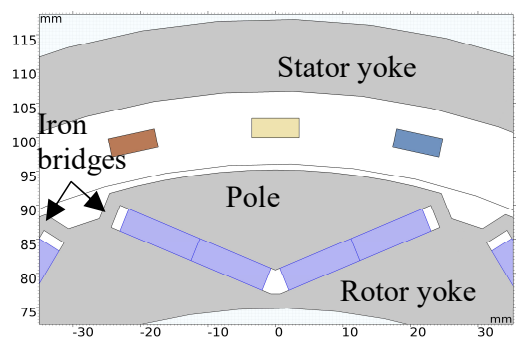

b)

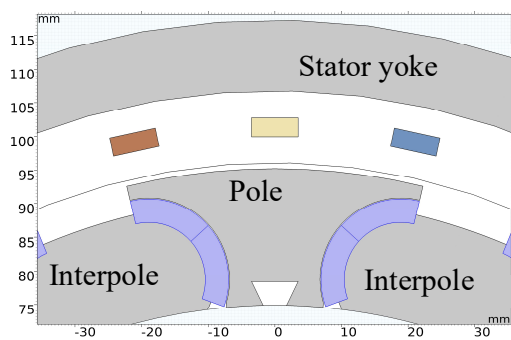

c)

Fig. 2 Classical geometries considered for the ASuMED demonstrator: surface mounted stacks a), interior mounted stacks b), and geometry finally developed, C-shape c).

TABLE II

MAGNETIC CHARACTERISTICS OF THE STACKS IN EACH GEOMETRY

\begin{tabular}{|l|c|c|c|c|c}
\hline Geometry of the stacks & $\hat{B}_{\text {rem }}(\mathrm{T})$ & $\begin{array}{c}\text { Flux per } \\
\text { pole }(\mathrm{Wb})\end{array}$ & $\begin{array}{c}\max \left(\Delta B_{\text {norm }}\right) \\
(\mathrm{mT})\end{array}$ & $\begin{array}{c}\max \left(\Delta B_{\text {tan }}\right) \\
(\mathrm{mT})\end{array}$ & $\begin{array}{c}\text { Stack length } \\
(\mathrm{mm})\end{array}$ \\
\hline Surface mounted & 1.35 & $1 \cdot 10^{-2}$ & 100 & 150 & 49.8 \\
\hline Interior mounted & 0.67 & $4.6 \cdot 10^{-3}$ & 25 & 20 & $2 \times 22.8$ \\
\hline C-shape & 0.91 & $6.9 \cdot 10^{-3}$ & 45 & 40 & $2 \times 25.2$ \\
\hline
\end{tabular}


These apparently incompatible requirements -surrounding the stacks with iron to protect them against the remaining airgap harmonics but at the same time avoiding leakage paths- prompted us to develop the C-shape configuration (Fig $2 \mathrm{c}$ )). Unlike permanent magnets, the high resistance at compression in the normal direction provided by the substrate allows the stacks to be used as structural element and the iron bridges responsible for most of the leakage in conventional configurations can be removed. In this case the interpole sections are locked in place by the stacks themselves and their load is transmitted to the poles, which protect the stacks from variations of the magnetic flux density during operation. This also favours magnetization through a low reluctance path in the $d$-axis direction (the centre of the pole) and conveys the mechanical loads to the internal part of the rotor (which is responsible for cooling), by a dovetail attachment. Furthermore, the length of the stacks, the active element of the rotor responsible for flux trapping, is maximized by using a curved shape, hence its name. This profile also allows for a larger pitch of the pole shoes, which increases saliency and shielding for the stacks, while providing the clearance necessary for the dovetail at their lower ends.

In this configuration the values of magnetic flux variation are roughly twice that of the buried stacks lay-out. However, they are still much smaller than those of surface mounted stacks, and while the remanent flux levels have improved in this configuration, they are still lower than conventional permanent magnets. Better magnetization methods must be developed in the future for this solution to be competitive.

In all these previous studies and in the rest of this work, field cooling magnetization has been assumed. This procedure can straightforwardly be applied to a fully superconducting motor with a double cryostat and distributed winding by cooling first the stator, then circulating a very high DC current in the $d$-axis of the now superconducting winding, then finally cooling the rotor. When the DC current in the stator is slowly decreased, currents are induced in the rotor stacks opposing to the reduction of the flux. Zero field cooling involves applying at least twice the intended trapped flux and thus requires more demanding conditions [17]. One variant of this later procedure, pulse magnetization, is especially widespread in laboratory environments to study samples and despite some preliminary research backing its use in motors [18], the actual application in an electrical machine remains problematic. Superconducting windings cannot be pulsed, suffering quenching when the critical current density is reached. To overcome this, especially devised coils must be installed in the stator of a fully superconducting machine. Furthermore, reproducibility of the flux level attained in one stack cannot be guaranteed [8]. In the project the field cooling option was chosen, a simpler solution for a machine of this small size.

\section{Finite Element Model}

Traditionally in the design of a radial electrical machine, its electromagnetic state is determined in just a crosssection. This greatly simplifies the Maxwell equations to be applied since in 2D and for the low frequencies they operate, only a scalar state variable needs to be solved, the component of the magnetic vector potential $\vec{A}$ perpendicular to that plane, $A_{z}$ :

$$
-\nabla \cdot\left(\frac{1}{\mu} \nabla A_{z}\right)+\sigma \frac{\partial A_{z}}{\partial t}=J_{z}
$$

where $\mu$ is the magnetic permeability, $\sigma$ the conductivity of the material and $J_{z}$ the current density parallel to $A_{z}$. The Coulomb gauge $\nabla \cdot \vec{A}=0$ is used to fully determine $\vec{A}$, whereas we can obtain the magnetic flux density $\vec{B}$ simply as its curl:

$$
\vec{B}=\nabla \times \vec{A}
$$

For such designing tasks, this $A$-formulation is completed by further numerical techniques that permit, for instance, accounting for the relative movement of rotor and stator, the computation of the yielded torque and the influence of $3 \mathrm{D}$ effects by means of lumped parameters in coupled current and voltage circuit equations. Overall, computer codes based in the $A$-formulation provide the necessary answers for the development of a standard conventional electrical machine [19].

Yet this well stablished procedure cannot be hastily extended to the study of devices with superconductive regions. Type II superconductors can carry elevated levels of current since microscopic conventional areas exist within them that provide a path for the magnetic flux lines to cross an otherwise diamagnetic material. The oscillations of these flux lines, simply by thermal activation, induce an electrical field whose interaction with the circulating current creates a dissipative effect [20]. This is taken into account by means of a finite, and highly non-linear, resistivity in the form [15]: 


$$
\rho(|\vec{J}|)=\frac{E_{c}}{J_{c}}\left(\frac{|\vec{J}|}{J_{c}}\right)^{n-1}
$$

with in the most general case the dependences $J_{c}=J_{c}(\vec{B}(\theta), T)$ and $n=n(|\vec{B}|)$. These values of the critical current $J_{c}$ are obtained by detailed material characterization essays at different values of temperature $T$, magnetic flux density $\vec{B}$ and angle of the flux $\theta \cdot \vec{J}$ accounts for the current density at that point and the threshold field $E_{c}$ is usually taken as $1 \mu \mathrm{V} / \mathrm{m}$. The evolution of the exponent $n$ is more difficult to parametrize, and usually comprises averaging several measurements under different conditions.

The insertion of an equivalent form of (4), with the electric field as variable, in (2) as the inverse of the conductivity $\sigma$ to assess the current distribution in a superconducting material creates numerical convergence problems, since this variable is unbounded at the origin (for the initialization value, $|\vec{E}|=0$, the conductivity $\sigma$ increases towards infinity) [15]. Some mitigation methods yielded results modelling superconductors with the $A$-formulation, but nowadays a more rigorous technique consists of employing the $H$-formulation instead, derived from the Faraday's and Ampere's laws in the form:

$$
\frac{\partial \mu \vec{H}}{\partial t}+\nabla \times \rho(\nabla \times \vec{H})=0
$$

here the direct use of (4) to obtain the current distribution in a superconductor provides a slow increase for small values of $\vec{J}=\left[J_{z}\right]$ such as the ones present in the superconductor at the beginning of the simulation. On the other hand, a vector quantity is to be solved $\vec{H}=\left[H_{x} H_{y}\right]^{T}$. Nevertheless, the addition of this formulation to the commercial finite element software COMSOL has popularized its use among the superconductor's community.

Surely the development of a combination of both forms to solve the Maxwell equations would benefit the study of different electrical machines topologies, providing an easier modelling and processing of the superconducting regions and, at the same time, allowing the utilization of the classic electromechanical principles to check the performance of the design. This would effectively erase the difference between superconducting and conventional motors at this phase of their development. Grilli et Al., within the ASuMED project, proposed and implemented in the aforementioned commercial software the weakly coupling of both formulations by a tramodel boundary [15]. This approach has been applied in this work to simulate the behavior of the novel rotor lay-out during magnetization and operation. The interformulation boundary is set in the airgap of the machine, within the rotor region, next to and parallel to the moving boundary between the stator and the rotor. In this manner the stator is modelled as a conventional one (but with much higher current densities in its winding) using the $A$-formulation, whereas the rotor, iron, stacks and a part of the airgap use the $H$-one. The relative movement between the rotor and stator meshes is coupled in the $A$-formulation, an easier procedure due to its scalar nodal values, as done in a conventional machine simulation.

Regarding the materials, silicon steel M270-35A has been assumed for the stator and rotor yokes, whose behavior at cryogenic temperatures is better understood compared to $\mathrm{Fe}-\mathrm{Co}$ alloys exhibiting higher magnetic saturation values, whereas the superconducting regions are considered filled with homogenized AMSC tape, which features a critical current of $391 \mathrm{~A} / \mathrm{cm}$-width at $77 \mathrm{~K}$ and self field and an engineering current density of $4.49 \cdot 10^{8} \mathrm{~A} / \mathrm{m}^{2}$. The substrate on which the superconductor is deposited comprises a Ni-5at\%W alloy with a yield stress of $257 \mathrm{MPa}$ [9]. The tape has a width of $46 \mathrm{~mm}$ and thickness of $87 \mu \mathrm{m}$, which means that a single tape immersed in liquid nitrogen can carry up to $1.8 \mathrm{kA}$ before quenching. This transport current value more than doubles at liquid hydrogen temperatures.

Consistently with the operation of the demonstrator, two stages are simulated by means of this combined formulation: an initialization consisting of the magnetization by field cooling of the stacks, and then motoring with losses computation. The variation of the magnetic flux density on the stacks during operation is also utilized to qualitatively assess their survivability against demagnetization, comparing it with experimental measurements. As mentioned previously, the field cooling process consists of applying a magnetic flux density on the stacks previously to reducing their temperature below the critical one (Fig. 3). DC currents in the stator supplied in the $d$-axis are used for this purpose. When these currents are slowly ramped down (Stage 3 in Fig. 3), currents are induced in the superconductor forming the stacks, effectively trapping a flux level that reproduces the one applied [21].

After the magnetization AC currents in the stator are ramped up and the rotor begins to turn when the maximum torque is reached (Fig. 4). The load angle for maximum torque is determined by time-harmonic simulations on a conventional model of the same motor geometry. 

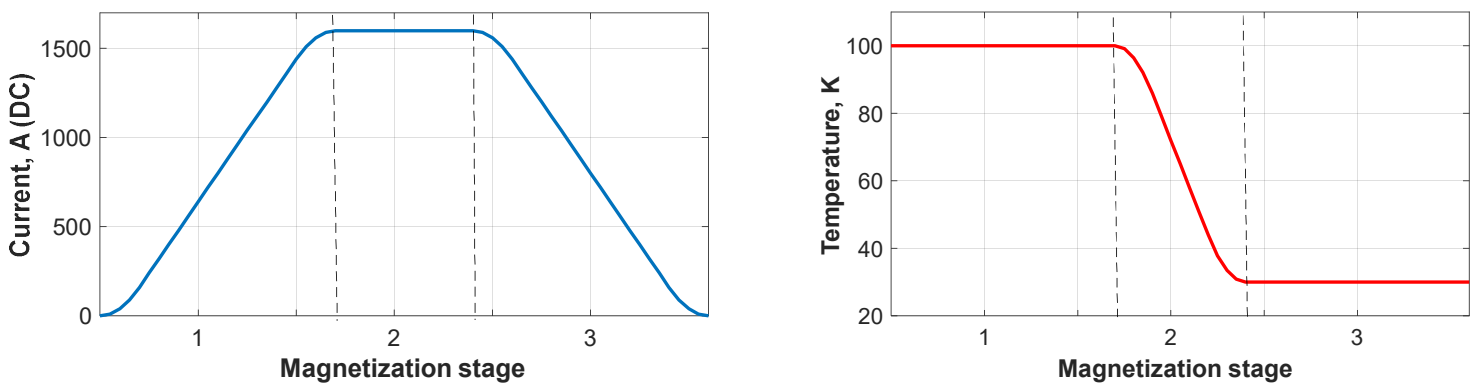

Fig. 3 DC current profile fed to the stator (left) and rotor temperature (right) during the field cooling magnetization process. Stages 2 and 3 would take several minutes to be completed.

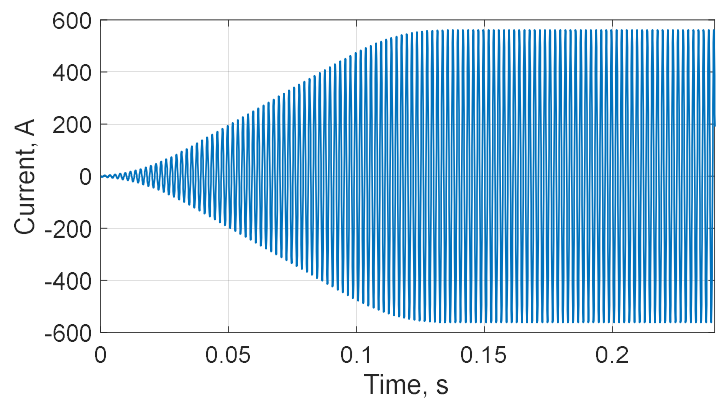

Fig. 4 Current in phase A during the motoring part of the simulation.

\section{Results}

This section is divided in two subsections, the first one comprises the results of the magnetization process and the second one focuses on the output of the motoring simulation, where the applicability of the proposed design: output torque, electromagnetic losses in the stacks and some considerations about the impact of the demagnetization in the operation of the machine, are examined.

\section{A. Magnetization}

The results of simulating the field cooling magnetization process on the motor architecture proposed in this work are shown in Fig. 5. The first diagram (Fig. 5 a) complements Fig. 3 as it presents the rising current in one stack, computed as half the value of the integral of $\left|J_{z}\right|$ in its cross section. The maximum value is $5.4 \mathrm{kA}$ with a standard deviation between stacks of $22 \mathrm{~A}$. This small difference can be attributed to numerical causes. The modest value of currents induced is motivated by the magnetization procedure and the big airgap resulting from the suppression of the stator teeth. The stack is thus widely undersaturated and, as seen in Fig. 5 b), this yields a trapezoidal shape of the magnetic flux density on its surface with heavy leakage at both sides, due to the rapidly descending currents at its edges. The value of the magnetic flux density in the middle of the stack is just $0.75 \mathrm{~T}$ and the average only reaches $0.64 \mathrm{~T}$, well below what would be achievable by conventional permanent magnets. The necessity of adding a clearance of $0.25 \mathrm{~mm}$ of non-ferromagnetic material around the stack for computational reasons accounts for the difference to the value expected from Table II in which conventional simulations with imposed-current density regions were used. Nevertheless, the flux per pole is $7 \cdot 10^{-3} \mathrm{~Wb}$, as calculated, due to the distinct current profile in the superconducting regions compared the one supposed for populating Table II (trapezoidal vs. triangular) and the different saturation patterns they create in the pole. 


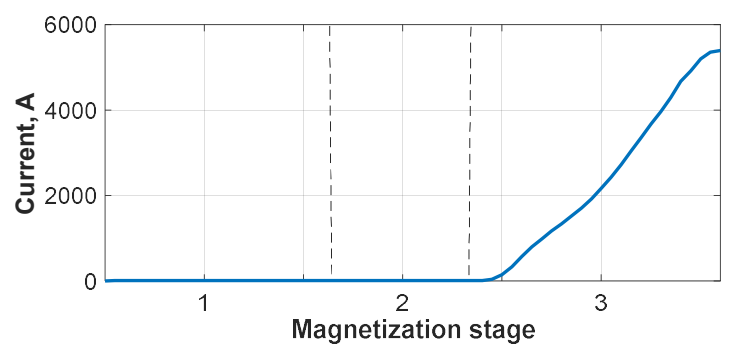

a)

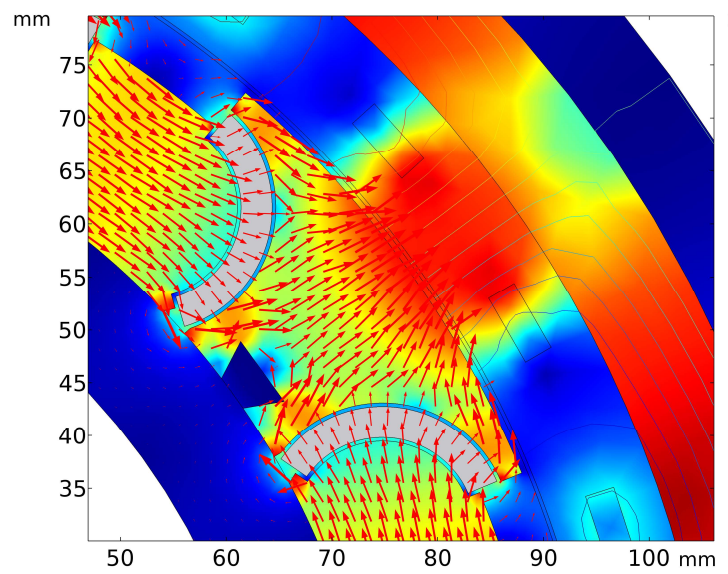

c)

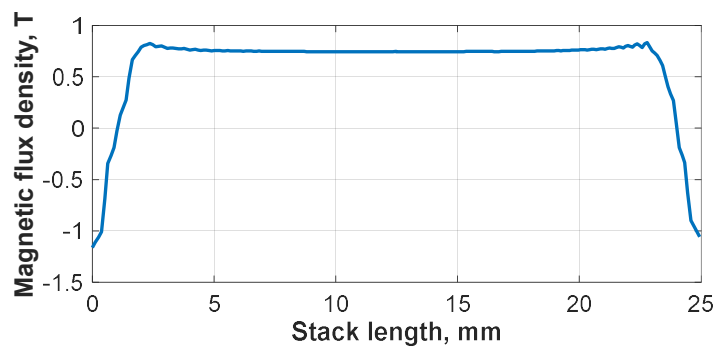

b)

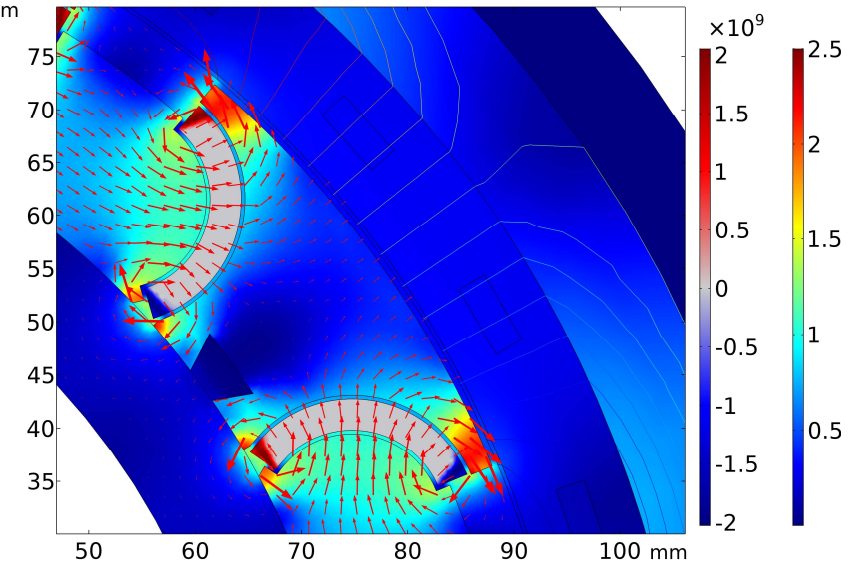

d)

Fig. 5 Rising current in the stack during the magnetization process, a); magnetic flux density profile at the pole-facing surface of the stack, showing leakage at its edges, b); and distribution of magnetic flux density

(rainbow scale in $\mathrm{T}$ ) and current density (red-blue scale in $\mathbf{A} / \mathbf{m}^{2}$ ) at the middle, c), and end, d), of the magnetization process for the proposed motor architecture. Arrows indicate the magnitude and direction of the magnetic flux density in the rotor.

Figs. 5 c) and d) present the distributions of current density in the stack and the magnetic flux density in the rest of the machine's cross section at the middle ("Stage 2" in Fig. 3 and 5a)) and at the end of the magnetization procedure. The effect of feeding the three phases with 1,600 A DC is seen in Fig. $5 \mathrm{c}$ ), with heavy magnetic saturation in the iron and no current in the stacks yet, since the temperature is above the critical value for the superconductor, $T_{c}$. In Fig. 5 d), the limited loading of the stacks is clearly depicted by the induced currents flowing just along its edges, in and out, perpendicular to the cross section, whereas the center remains empty. Leakage at their edges is limited by the saturation of iron at the pole tips and bottom, but in any case this effect is very significant, since it must accounted twice, during the magnetization, the magnetic flux induced by the stator coils skips the stacks, and afterwards, the big airgap chosen for this design implies this flux inverts its sense in those areas, creating a significant return, although always a lower amount compared to what would happen using traditional configurations of interior mounted permanent magnets. Counterintuitively, thinner stacks do not improve the performance of the machine, since the more saturated they are, the more triangular the magnetic flux density profile they produce, which exacerbates this fringe leakage.

\section{B. Motoring}

Similarly to conventional machine's studies, in which a time-harmonic simulation is used to obtain initial values for the time-stepping calculations, in this work we have initialized the motoring simulation with the results of last time-step from the magnetization process, which reproduces the actual operational procedure to be followed in the demonstrator. The magnetic flux distribution in the cross-section of the machine after magnetization is shown in Fig. 6 a) and during operation in Fig. 6 b). The effect of the stator currents is clearly appreciated, heavily saturating the forward tip of the pole and increasing the leakage from the interpole region. Fig $6 \mathrm{c}$ ), which corresponds to Fig. 5d) 
under motoring conditions, clearly portrays the distortion of the currents flowing in the stack below this tip due to saturation.

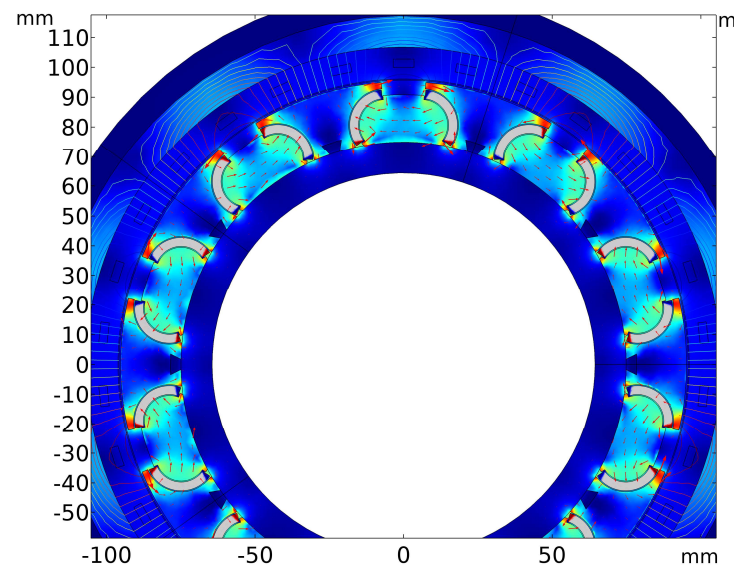

a)

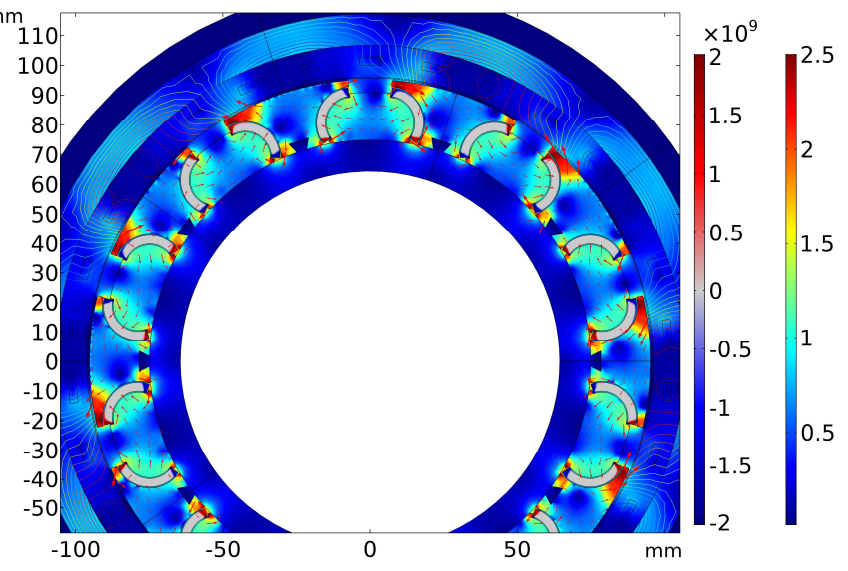

b)

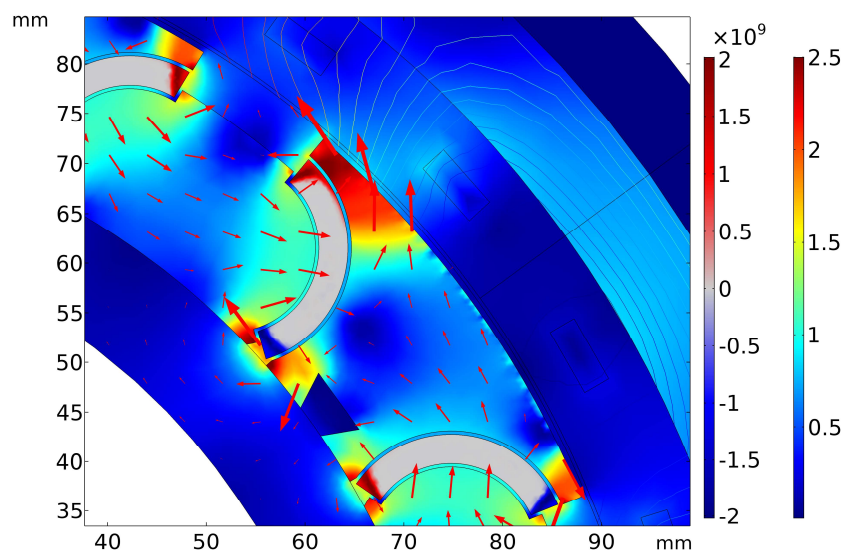

c)

Fig. 6 Magnetic flux density (rainbow scale, in T) and current density in the stacks (red-blue scale, in A/m²) in the motor after magnetization a), and during operation, b) and c).

\section{Torque}

The profile of the average torque during the simulation can be seen in Fig. 7. During the first instants of the simulation shown here, the rotor is still whilst the stator is being energized. $15 \mathrm{~ms}$ later, at the top of the torque amplitude sinusoid, rotation begins. The transient ends at $0.13 \mathrm{~s}$, following constant operation at around $500 \mathrm{~N} \cdot \mathrm{m}$, that is, yielding a power of $314 \mathrm{~kW}$ with an approximate power density above $18 \mathrm{~kW} / \mathrm{kg}$, not considering anything else but the motor's electromagnetic active parts. The slow trend appreciated in Fig. 7 afterwards is the effect of the settling of the currents in the rotor stacks. 


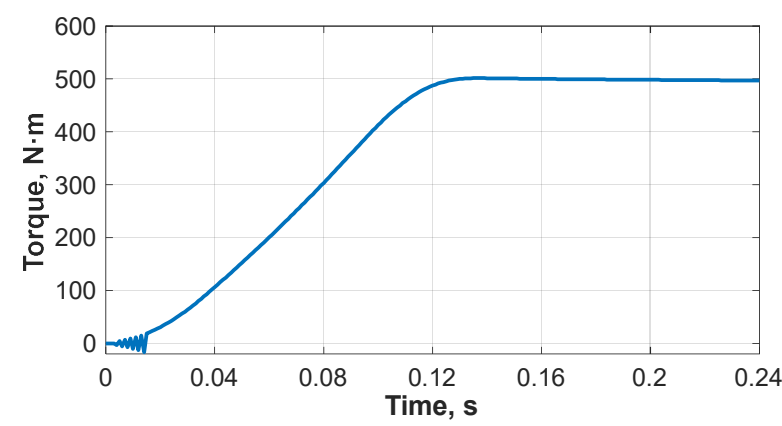

Fig. 7 Torque level yielded by the proposed design as stator currents are ramped up and then kept constant (beyond $0.13 \mathrm{~s}$ ). Rotation commences at $15 \mathrm{~ms}$.

\section{Losses in the stacks}

From the 2D model, the electromagnetic losses in each stack can be computed from the numerical solution simply as the Joule heating in the superconducting region times the active length of the machine $L_{M}[22]$ :

$$
P_{\text {loss }}=L_{M} \int_{\text {stack }} \vec{E} \cdot \vec{J} d S
$$

The average result of applying this equation to the 16 stacks is shown in Fig. 8. It is noticeable that electromagnetic transient produced by ramping up the stator currents increases the losses in the stack sharply, more than 5 times the ones expected for steady state, in this rather severe evolution: from zero to full torque in $0.13 \mathrm{~s}$. This might have its impact if the motor is expected to be used for steering or attitude control, with continuous and rapid variations of the commanded power.

On the other hand, the total amount of losses remains in manageable levels for the cooling system, below $100 \mathrm{~W}$ in any case for the full rotor, and much less, around $13 \mathrm{~W}$ for steady state [16].

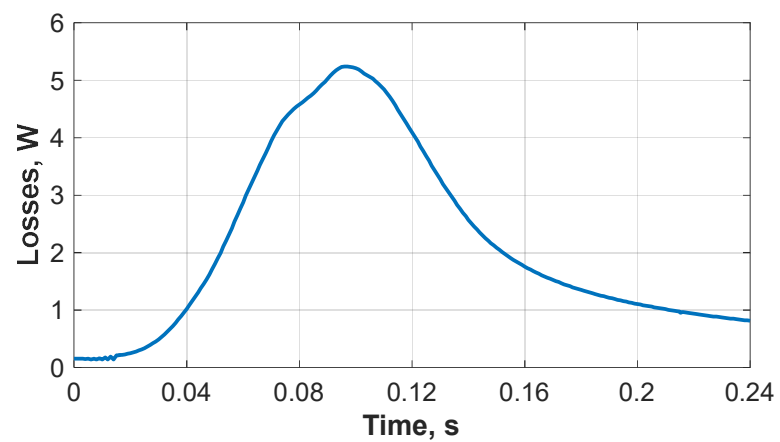

Fig. 8 Losses in a stack during the operation of the motor.

\section{Demagnetization}

As stated previously, the macroscopic nature of the currents circulating in the stacks is translated to the fact that any variation of the magnetic flux density will influence them. Cross-fields stir the current vortexes breaking their pattern and effectively demagnetizing the superconductor [11]. Fig. 9 shows the result of a laboratory test using an actual rotating electrical machine in which a 9 insulated layer $(<1 \mathrm{~mm})$ AMSC stack was fixed to the surface of the rotor, magnetized and then rotated in a liquid nitrogen environment $(77 \mathrm{~K})$ at $10 \mathrm{~Hz}$. As a cross-field estimated in 25 $\mathrm{mT}$ is applied, the peak back-emf measured in the stator (which for $1 \mathrm{~V}$ roughly corresponds to $100 \mathrm{mT}$ trapped in the stack) portrays a two stage evolution, sharply decreasing in the first seconds, followed by a slow decay [23]. More than $40 \%$ of the initial magnetization is rapidly lost, despite the relatively small cross-field, but on the other hand the remaining lasts for several minutes.

The cross-field levels obtained from the numerical simulations using the combined formulation decrease the values obtained with the conventional model for Table II due to the saturation of the poles, showing a variation of the 
magnetic flux density of around $10 \mathrm{mT}$ in side of the stack facing the airgap (for a trapped field of $0.64 \mathrm{~T}$ ) during steady conditions at full load. Therefore, some demagnetization is expected to occur, especially during transients, however, there should be enough flux to perform the planned tests for the demonstrator, provided the evolution is similar to the one measured in Fig. 9.

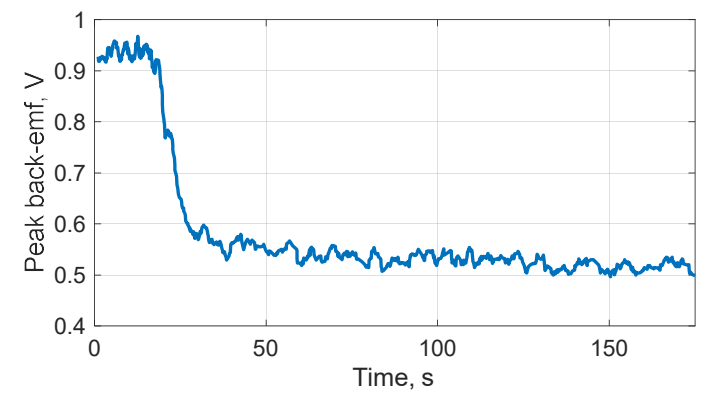

Fig. 9 Decreasing peak back-emf induced by a rotating stack in the airgap of an electrical machine as a crossfield is applied around $t=20 \mathrm{~s}$ and maintained thereafter.

\section{Conclusion}

This work presents the results of simulating a novel rotor architecture, specifically developed for using stacks of superconductive tape, by a new computational approach that allows modelling the behavior of superconducting regions during the operation of a rotating electrical machine. The procedure permits initializing the state of the machine in realistic conditions and obtaining its performance characteristics, such as torque, electromagnetic losses in the stacks and some insight in their demagnetization rate. According to these results, a fully superconducting motor based on stacks is feasible, although several problems persist, such as a modest trapped flux, flux leakage around the stacks and some level of demagnetization. Nevertheless, the novel rotor lay-out reduces their influence and offers plenty of room for improvement. It remains the task of confirming these results with experimental tests once the demonstrator becomes operational.

\section{Acknowledgments}

This research is financially supported by the European Union's Horizon 2020 research innovation programme under grant agreement No 7231119 (ASuMED consortium) and EPSRC grant EP/P000738/1.

\section{References}

[1] Felder, J. L., Kim, H. D., Brown, G. V., "Turboelectric Distributed Propulsion Engine Cycle Analysis for Hybrid-Wing-Body Aircraft," 47th AIAA Aerospace Sciences Meeting including The New Horizons Forum and Aerospace Exposition, Orlando, Florida, 2009.

[2] Felder, J. L., Kim, H. D., Brown, G. V., Chu, J., “An Examination of the Effect of Boundary Layer Ingestion on Turboelectric Distributed Propulsion Systems," 49th AIAA Aerospace Sciences Meeting including The New Horizons Forum and Aerospace Exposition, Orlando, Florida, 2011.

[3] Aerobatic Airplane "Extra 330LE" with world-record electric motor from Siemens [online], https://www.siemens.com/press/pool/de/events/2016/corporate/2016-12-innovation/inno2016-aerobatic-airplane-e.pdf

[4] Pyrhönen, J., Jokinen, T. and Hrabovcová, V., Design of Rotating Electrical Machines, 2nd ed. New York, NY, USA: Wiley, 2014.

[5] Epstein, A. H., O’Flarity, S. M., "Considerations for Reducing Aviation's CO2 with Aircraft Electric Propulsion”, AIAA Journal of Propulsion and Power, Vol. 35, No. 3 May-Jun. 2019, pp. 572-582.

[6] Kalsi, S. S., Weeber, K., Takesue, H., Lewis, C., Neumueller, H.-W., Blaugher, R. D., "Development Status of Rotating Machines Employing Superconducting Field Windings," Proc. IEEE, Vol. 92, No. 10, Oct. 2004, pp. 1688-1704.

[7] ASuMED project website: https://cordis.europa.eu/project/rcn/209910/factsheet/en

[8] Shengnan Zou, Magnetization of High Temperature Superconducting Trapped-Field Magnets, PhD Thesis, KIT Scientific Publishing, 2017. (Available on-line: https://publikationen.bibliothek.kit.edu/1000073152 ).

[9] Patel, A., Baskys, A., Mitchell-Williams, T., McCaul, A., Coniglio, W., Hänisch, J., Lao, M., Glowacki, B. A., "A trapped field of 17.7 T in a stack of high temperature superconducting tape," Superconductor Science and Technology, Vol. 31 No. 9, 2018, 09LT01. 
[10] Iwasa, Y., Case Studies in Superconducting Magnets, Design and Operational Issues, Second edition, Springer, 2009.

[11] Campbell, A., Baghdadi, M., Patel, A., Zhou, D., Huang, K. Y., Shi, Y., Coombs, T., "Demagnetisation by crossed fields in superconductors," Superconductor Science and Technology, Vol. 30, No. 3, 2017, 034005.

[12] Ahn, J., Han, C., Kim, C., Choi, J., "Rotor Design of High-Speed Permanent Magnet Synchronous Motors Considering Rotor Magnet and Sleeve Materials," IEEE Trans. Appl. Supercond., Vol. 28 No. 3, Apr. 2018, 5201504.

[13] Rao, J., Ronghai, Q., Jimin, M., Xu, W., "Investigate the influence of magnetic bridge design on mechanical strength and electromagnetic characteristics in high speed IPM machines," 17th International Conference on Electrical Machines and Systems (ICEMS), 22-25 Oct. 2014

[14] Patel, A., Climente-Alarcon, V., Baskys, A., Glowacki, B. A., Reis, T., "Design considerations for fully superconducting synchronous motors aimed at future electric aircraft," IEEE 5th International Conference on Electrical Systems for Aircraft, Railroad, Ship Propulsion and Road Vehicles and International Transportation Electrification Conference, Nottingham, UK, 2018

[15] Brambilla, R., Grilli, F., Martini, L., Bocchi, M., Angeli, G., "A finite-element method framework for modelling rotating machines with superconducting windings," IEEE Transactions on Applied Superconductivity, Vol. 28, No. 5, Aug. 2018, 5207511.

[16] Perez, A., van der Woude, R. R., Dekker, R., "Rotor Cooling Concept for the ASuMED Superconductive Motor," IOP Conf. Ser.: Mater. Sci. Eng. 5020121392019.

[17] Climente-Alarcon, V., Patel, A., Baskys, A., Glowacki, B. A., "Design considerations for electric motors using stacks of high temperature superconducting tape as permanent magnets," IOP Conf. Ser.: Mater. Sci. Eng. 5020121822019.

[18] Zhang, M., “A new world record for superconducting trapped field magnet," Superconductor Science and Technology, 2019. (in press, https://doi.org/10.1088/1361-6668/ab17e)

[19] Arkkio, A., Analysis of Induction Motors Based on the Numerical Solution of the Magnetic Field and Circuit Equations, PhD. Thesis, 1986, Aalto University, Finland. (on-line: http://lib.tkk.fi/Diss/198X/isbn951226076X/)

[20] Kim, Y. B., Hempstead, C. F., Strnad, A. R., "Flux-flow resistance in Type-II superconductors," Physical Review, Vol. 139, No. 4A, Aug. 1965, pp. 1163-1172.

[21] Climente Alarcon, V., Patel, A., Baskys, A., Glowacki, B. A., "Computation of Superconducting Stacks Magnetization in an Electrical Machine," IEEE Transactions on Applied Superconductivity, (accepted for publication, doi: 10.1109/TASC.2019.2923537).

[22] Rhyner, J., "Magnetic properties and AC-losses of superconductors with power law current-voltage characteristics," Physica C, Vol. 212, Jul. 1993, pp. 292-300.

[23] Smara, A., Mineev, N., Climente-Alarcon, V., Patel A., Baskys, A., Glowacki, B. A., Reis, T., "Experimental Assessment of Rotor Superconducting Stack Demagnetization in $\mathrm{LN}_{2}$ Environment," Superconductor Science and Technology, Vol. 32, No. 8, 085009, 2019. 